\title{
Intrinsic concentration dependences in the HgCdTe quantum well in the range of the insulator-semimetal topological transition
}

\author{
E.O. Melezhik ${ }^{1}$, J.V. Gumenjuk-Sichevska ${ }^{2}$, S.A. Dvoretskii ${ }^{3}$ \\ ${ }^{1} V$. Lashkaryov Institute of Semiconductor Physics, NAS of Ukraine \\ 41, prospect Nauky, 03028 Kyiv, Ukraine \\ Corresponding author e-mail: emelezhik@gmail.com; e-mail gumenjuk@gmail.com \\ ${ }^{2}$ Institute of Semiconductor Physics of SB RAS, \\ 13, pr. Lavrentieva, 630090 Novosibirsk, Russia
}

\begin{abstract}
The band structure and dependences of the intrinsic concentration in the mercury-cadmium-telluride (MCT) $\mathrm{Hg}_{0.32} \mathrm{Cd}_{0.68} \mathrm{Te} / \mathrm{Hg}_{1-\mathrm{x}} \mathrm{Cd}_{\mathrm{x}} \mathrm{Te} / \mathrm{Hg}_{0.32} \mathrm{Cd}_{0.68} \mathrm{Te}$ quantum wells in the framework of the $8 \times 8$ k.p envelope function method on the well width $L$ and composition $x$ were calculated. Modeling of the energy spectra showed that the intrinsic concentration can vary about an order of magnitude with variation of the well width and chemical composition in the range of $x \leq 0.16$ and well width $L \leq 20 \mathrm{~nm}$ at the liquid nitrogen temperature. These strong variations of the carrier concentration are caused by the insulator-semimetal topological transition.
\end{abstract}

Keywords: mercury cadmium telluride $\mathrm{HgCdTe}$, quantum well, insulator-semimetal topological transition, hot electron bolometer.

Manuscript received 17.01.14; revised version received 25.04.14; accepted for publication 12.06.14; published online 30.06.14.

\section{Introduction}

Recent studies [1] have shown that mercury cadmium telluride (MCT) can be promising for the development of $\mathrm{THz} / \mathrm{sub}-\mathrm{THz}$ detectors operating at moderate cooling. Whereas MCT bolometer detector noise equivalent power (NEP) is sufficiently dependent on the intrinsic concentration value [2]. We aimed to find proper conditions to reach better NEP conditions for the MCT bolometric structure based on quantum well. The purpose of our investigation was to outline the chemical composition of QW and its width in the insulatorsemimetal topological transition region to choose their proper values for growing such structures as $\mathrm{THz} / \mathrm{sub}$ $\mathrm{THz}$ detectors.

The insulator-semimetal topological transition in $\mathrm{CdTe} / \mathrm{HgTe} / \mathrm{CdTe}$ quantum wells takes place at the well thickness $L \approx 8 \mathrm{~nm}$ and temperature $T=77 \mathrm{~K}$ [3]. At $L<$ $8 \mathrm{~nm}$, the band order in the well and inside barriers is equal, and at $L>8 \mathrm{~nm}$ the band structure of the well is inverted and the conduction band has $\Gamma_{8}$ symmetry. In our work, we investigate the topological transition conditions for the nonzero composition parameter $x$ in the well and inside barriers.

Calculations of the band structure and intrinsic concentrations were made at the temperature of liquid nitrogen $(T=77 \mathrm{~K})$ where these structures can be efficiently applied as $\mathrm{THz} / \mathrm{sub}-\mathrm{THz}$ detectors.

The energy spectrum of $\mathrm{Hg}_{0.32} \mathrm{Cd}_{0.68} \mathrm{Te} / \mathrm{Hg}_{1-\mathrm{x}} \mathrm{Cd}_{\mathrm{x}} \mathrm{Te}$ quantum wells was modeled. The composition $x \approx 0.68$ of the barrier layer was chosen, as it is often used in real heterostructures [4-6] to minimize misfit strains between the well and barrier layers. Moreover, in real structures there can be two sources of strains: from the lattice misfit between the well and barrier layer as well as from the lattice misfit between the barrier and substrate. These strains are often of opposite sign, and the resulting strain in the well is small. Since the strains can be eliminated technologically, they are neglected in numerical modeling. To account precisely these complex strains is a rather complicated procedure.

The calculations of energy spectra are provided in the framework of the envelope functions approach, when the carrier wave-function is expanded on the basis of 
eight Bloch band-edge (in-plane $k=0$ ) functions [7]. The system is assumed to be periodical, the barrier well width is chosen to correspond to the isolated quantum well. In the calculations, there was used the barrier of 100-nm width, which is good approximation for the infinite width barrier. The calculations were made for the [001] oriented quantum well, thus, the $z$-axis coincides with the growth direction of the well.

The carrier wave-function can be written as:

$$
\psi(r)=\sum_{n} F_{n}(r) u_{n}(r),
$$

where $F_{n}(r)$ are the envelope functions, $n$ varies from 1 up to 8 . Assuming translation invariance in the plane perpendicular to the growth direction (z-axis), $F_{n}(r)$ can be represented as:

$$
F_{n}(r)=\exp \left[i\left(k_{x} x+k_{y} y\right)\right] f_{n}(z) \text {. }
$$

The functions $f_{n}(z)$ in the well (A) and inside the barrier (B) are expanded on the full basis of the Legendre polynomials:

$$
\begin{aligned}
& f_{n}^{A}(z)=\sum_{i=1}^{\infty} c_{n a}^{i} L^{i}\left(\frac{2 z}{L}\right) \approx \sum_{i=1}^{i_{0}} c_{n a}^{i} L^{i}\left(\frac{2 z}{L}\right), \\
& f_{n}^{B}(z)=\sum_{i=1}^{\infty} c_{n b}^{i} L^{i}\left(\frac{2 z}{L_{B}}\right) \approx \sum_{i=1}^{i_{0}} c_{n b}^{i} L^{i}\left(\frac{2 z}{L_{B}}\right) .
\end{aligned}
$$

where $L_{i}$ are the Legendre polynomials of $i$-th order. The number $i_{0}$ of Legendre polynomials in the expansion (3), necessary to obtain accurate solutions, is taken to be 16 [7].

Energy levels and envelope-function coefficients can be found from the simultaneous solution of the system of coupled differential equations - Hamiltonian equation and boundary conditions system [7].

The Hamiltonian of the system in the 8-band basis is written as [7]: where $k_{\|}^{2}=k_{x}^{2}+k_{y}^{2}$ is the in-plane momentum,

$$
\begin{aligned}
& k_{ \pm}=k_{x} \pm i k_{y}, k_{z}=-\frac{i \partial}{\partial z}, \\
& T=E_{c}(z)+\frac{\hbar^{2}}{2 m_{0}}\left[(2 F+1) k_{\|}^{2}+k_{z}(2 F+1) k_{z}\right], \\
& U=E_{v}(z)-\frac{\hbar^{2}}{2 m_{0}}\left(\gamma_{1} k_{\|}^{2}+k_{z} \gamma_{1} k_{z}\right), \\
& V=-\frac{\hbar^{2}}{2 m_{0}}\left(\gamma_{1} k_{\|}^{2}-2 k_{z} \gamma_{2} k_{z}\right), \\
& R=-\frac{\hbar^{2}}{2 m_{0}}\left(\sqrt{3} \mu k_{+}^{2}-\sqrt{3} \bar{\gamma} k_{-}^{2}\right), \\
& \bar{S}_{ \pm}=-\frac{\hbar^{2}}{2 m_{0}} \sqrt{3} k_{ \pm}\left(\left\{\gamma_{3}, k_{z}\right\}+\left[\kappa, k_{z}\right]\right), \\
& \tilde{S}_{ \pm}=-\frac{\hbar^{2}}{2 m_{0}} \sqrt{3} k_{ \pm}\left(\left\{\gamma_{3}, k_{z}\right\}-\frac{1}{3}\left[\kappa, k_{z}\right]\right), \\
& C=\frac{\hbar^{2}}{m_{0}} k_{-}\left[\kappa, k_{z}\right] .
\end{aligned}
$$

Here, $[A, B]=A B-B A$ is the usual commutator, while $\{A, B\}=A B+B A$ is the anti-commutator for two operators $A$ and $B ; P$ is the Kane matrix element, $P$, as a rule, is taken to be equal in the well and inside the barrier (see e.g. [7]). $E_{c}(z)$ and $E_{v}(z)$ are the respective edges of the conduction and valence bands; $\Delta$ is the spin-orbit splitting. The Luttinger parameters $\gamma_{1}, \gamma_{2}, \gamma_{3}, \kappa$ and $F$ describe coupling with remote bands, the additional parameters $\mu$ and $\bar{\gamma}$ are defined as $\mu=\left(\gamma_{3}-\gamma_{2}\right) / 2, \quad \bar{\gamma}=\left(\gamma_{3}+\gamma_{2}\right) / 2$ [7]. The Luttinger parameters of $\mathrm{HgTe}$ and CdTe were taken from [7], they were assumed to vary linearly with concentration. As a rule, they are assumed to be temperature-independent.

$$
H=\left(\begin{array}{cccccccc}
T & 0 & -\frac{1}{\sqrt{2}} P k_{+} & \sqrt{\frac{2}{3}} P k_{z} & \frac{1}{\sqrt{6}} P k_{-} & 0 & -\frac{1}{\sqrt{3}} P k_{z} & -\frac{1}{\sqrt{3}} P k_{-} \\
0 & T & 0 & -\frac{1}{\sqrt{6}} P k_{+} & \sqrt{\frac{2}{3}} P k_{z} & \frac{1}{\sqrt{2}} P k_{-} & -\frac{1}{\sqrt{3}} P k_{+} & \frac{1}{\sqrt{3}} P k_{z} \\
-\frac{1}{\sqrt{2}} k_{-} P & 0 & U+V & -\bar{S}_{-} & R & 0 & \frac{1}{\sqrt{2}} \bar{S}_{-} & -\sqrt{2} R \\
\sqrt{\frac{2}{3}} k_{z} P & -\frac{1}{\sqrt{6}} k_{-} P & -\bar{S}_{-}^{\dagger} & U-V & C & R & \sqrt{2} V & -\sqrt{\frac{2}{3}} \widetilde{S}_{-} \\
\frac{1}{\sqrt{6}} k_{+} P & \sqrt{\frac{2}{3}} k_{z} P & R^{\dagger} & C^{\dagger} & U-V & \bar{S}_{+}^{\dagger} & -\sqrt{\frac{3}{2}} \widetilde{S}_{+} & -\sqrt{2} V \\
0 & \frac{1}{\sqrt{2}} k_{+} P & 0 & R^{\dagger} & \bar{S}_{+} & U+V & \sqrt{2} R^{\dagger} & \frac{1}{\sqrt{2}} \bar{S}_{+} \\
-\frac{1}{\sqrt{3}} k_{z} P & -\frac{1}{\sqrt{3}} k_{-} P & \frac{1}{\sqrt{2}} \bar{S}_{-}^{\dagger} & \sqrt{2} V & -\sqrt{\frac{3}{2}} \widetilde{S}_{+}^{\uparrow} & \sqrt{2} R & U-\Delta & C \\
-\frac{1}{\sqrt{3}} k_{+} P & \frac{1}{\sqrt{3}} k_{z} P & -\sqrt{2} R^{\dagger} & -\sqrt{\frac{3}{2}} \tilde{S}_{-}^{\dagger} & -\sqrt{2} V & \frac{1}{\sqrt{2}} \bar{S}_{+}^{\dagger} & C^{\dagger} & U-\Delta
\end{array}\right)
$$


Boundary conditions appear due to two physical requirements. First, the probability density to find electron near the defined coordinate should be continuous at the interface. It imposes the requirement of continuity of the wave-function (1). Band-edge Bloch functions are linearly independent, in-plane momentum is conserved when crossing the interface. Thus, the requirement of continuity of the wave-function (1) is equal to the requirement of continuity of each of the envelope wave-functions (3).

Second, the Luttinger parameters $\gamma_{1}, \gamma_{2}, \gamma_{3}, \kappa$ and $F$ are different in the well and inside the barrier. They are assumed to change its step-like character at the interface [7]. In the Hamiltonian (4), these parameters are used inside the $\frac{\partial}{\partial z}$ operator. Consequently, at the interface, one can face the situation when the functions inside $\frac{\partial}{\partial z}$ also change step-like character. Applying $\frac{\partial}{\partial z}$ to the step-like function leads to the infinite Hamiltonian matrix elements at the interface, which is certainly unphysical.

Thus, the second set of the boundary conditions requires continuity of all the equations inside the $\frac{\partial}{\partial z}$ operators. It can be written mathematically as the requirement of continuity of the integrals from each element of Eq. (4) over the $z$-coordinate. It is written as the requirement of continuity of the matrix product $D \cdot \bar{f}_{n}$ at the interface: where elements containing $P$ vanish because $P$ is taken to be the same in the well and inside the barriers. Here,

$$
\begin{aligned}
& t=-\frac{\hbar^{2}}{2 m_{0}}(2 F+1) \frac{\partial}{\partial z}, u=\frac{\hbar^{2}}{2 m_{0}} \gamma_{1} \frac{\partial}{\partial z}, \\
& v=-\frac{\hbar^{2}}{m_{0}} \gamma_{2} \frac{\partial}{\partial z}, \\
& \bar{s}_{ \pm}=\frac{\hbar^{2}}{2 m_{0}} i \sqrt{3}\left(\gamma_{3}-\kappa\right) k_{ \pm}, \\
& \bar{s}_{ \pm}^{\prime}=\frac{\hbar^{2}}{2 m_{0}} i \sqrt{3}\left(-\gamma_{3}+\kappa\right) k_{ \pm}, \\
& \tilde{s}_{ \pm}=\frac{\hbar^{2}}{2 m_{0}} i \sqrt{3}\left(\gamma_{3}+\kappa / 3\right) k_{ \pm}, \\
& \tilde{s}_{ \pm}^{\prime}=\frac{\hbar^{2}}{2 m_{0}} i \sqrt{3}\left(-\gamma_{3}-\kappa / 3\right) k_{ \pm}, \\
& c=\frac{\hbar^{2}}{m_{0}} i \kappa k_{-} .
\end{aligned}
$$

\section{Results}

The intrinsic concentrations and Fermi level were calculated by fitting the concentrations of electrons and heavy holes. The calculations are separated in two parts: (i) dependence of the carrier concentration on the well width and (ii) dependence of the carrier concentration on the composition $x$ of the well.

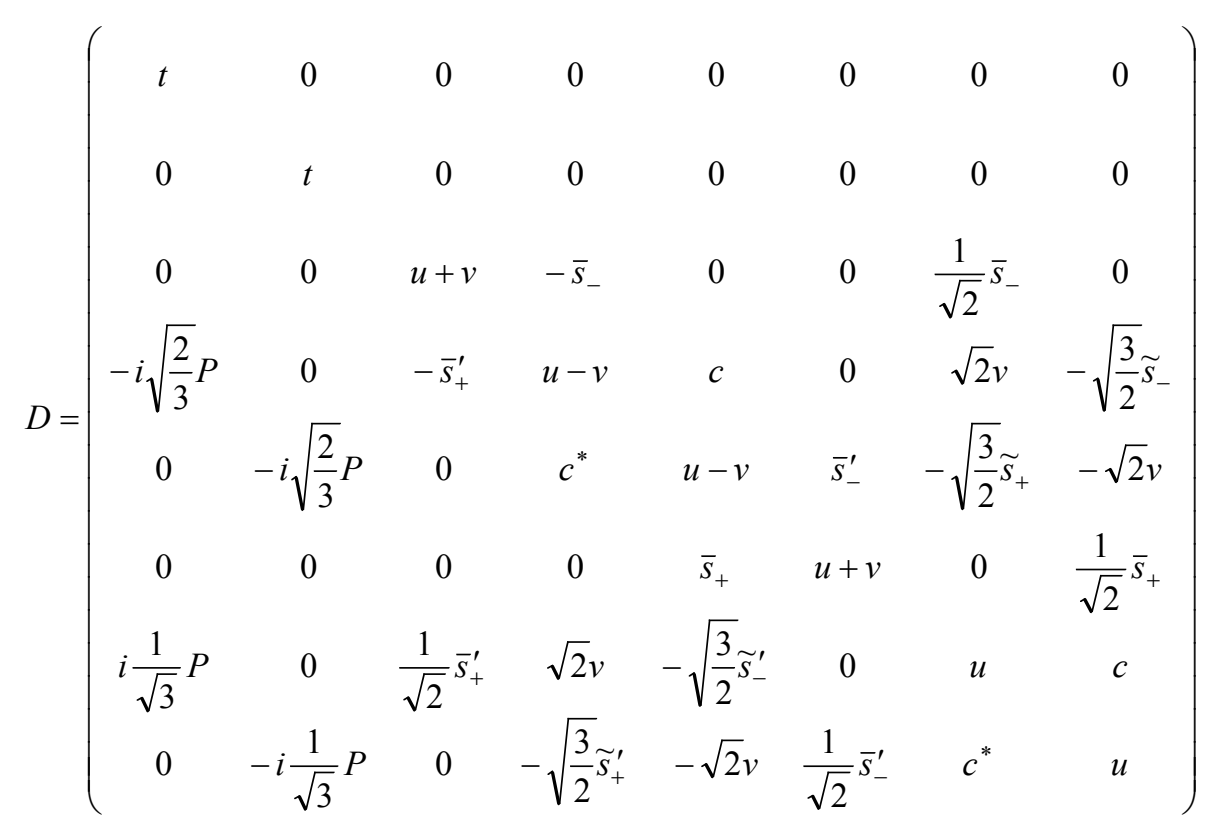




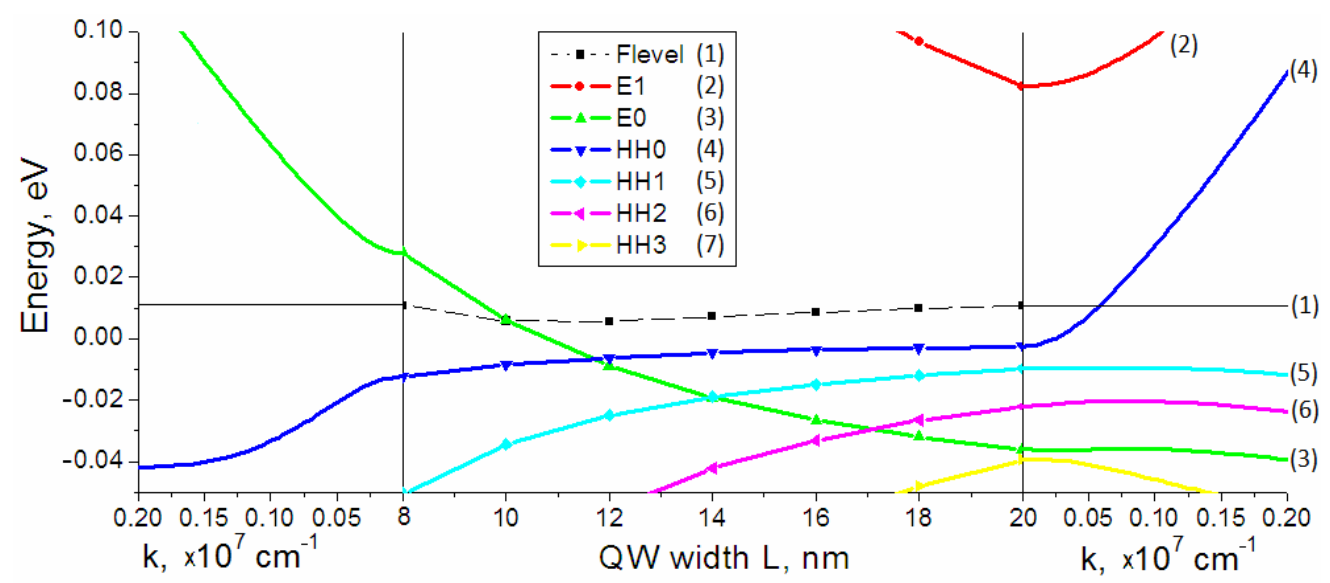

Fig. 1. Dependence of energy spectrum and Fermi level of QW with the composition $x=0.06$ on the well width $L$. Curves on the graph are marked as follows: Fermi level (1), E1 level - (2), E0 level - (3), HH0 level - (4), HH1 level - (5), HH2 level (6), HH3 level - (7).

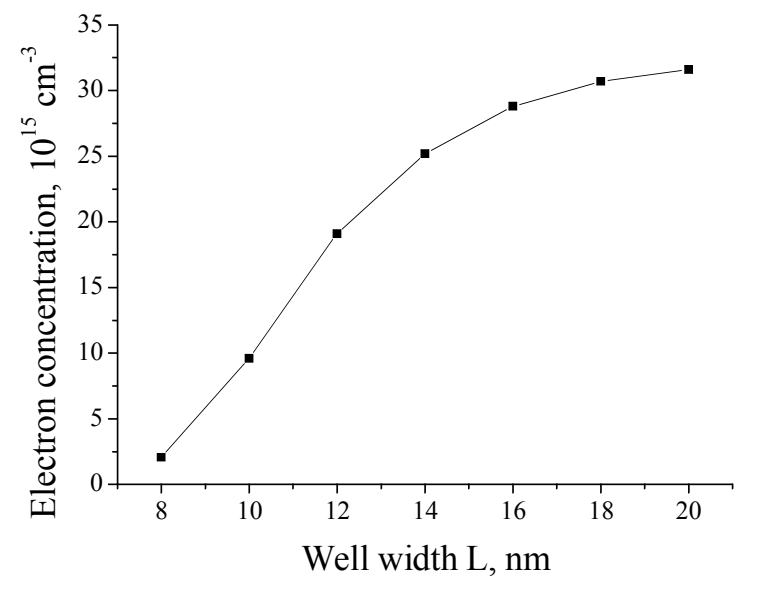

Fig. 2. Dependence of intrinsic concentration on the well width $L$ in QW with the composition $x=0.06$.
The dependence of the carrier concentration on $L$ (Fig. 2) is calculated for the composition of the well $x=0.06$. For this composition, the critical thickness of the insulator-semimetal topological transition is around $L=12 \mathrm{~nm}$ (see Fig. 1) and its influence on the intrinsic concentration can be examined.

From Fig. 2, one can see that the localized intrinsic concentration grows along with the well width. This growth is explained by the presence of the topological phase transition, which opens the band gap for widths less than $12 \mathrm{~nm}$. For larger well widths, the system is semimetal and the band gap is absent, thus, the intrinsic concentration demonstrates plateau at larger well widths.

Another important feature is that the local minimum of the Fermi level is situated near the topological transition.

One should note that for higher concentrations like $x=0.12$, the critical thickness is around $L \sim 20 \mathrm{~nm}$, and the growing section in Fig. 2 will be shifted to higher well widths.

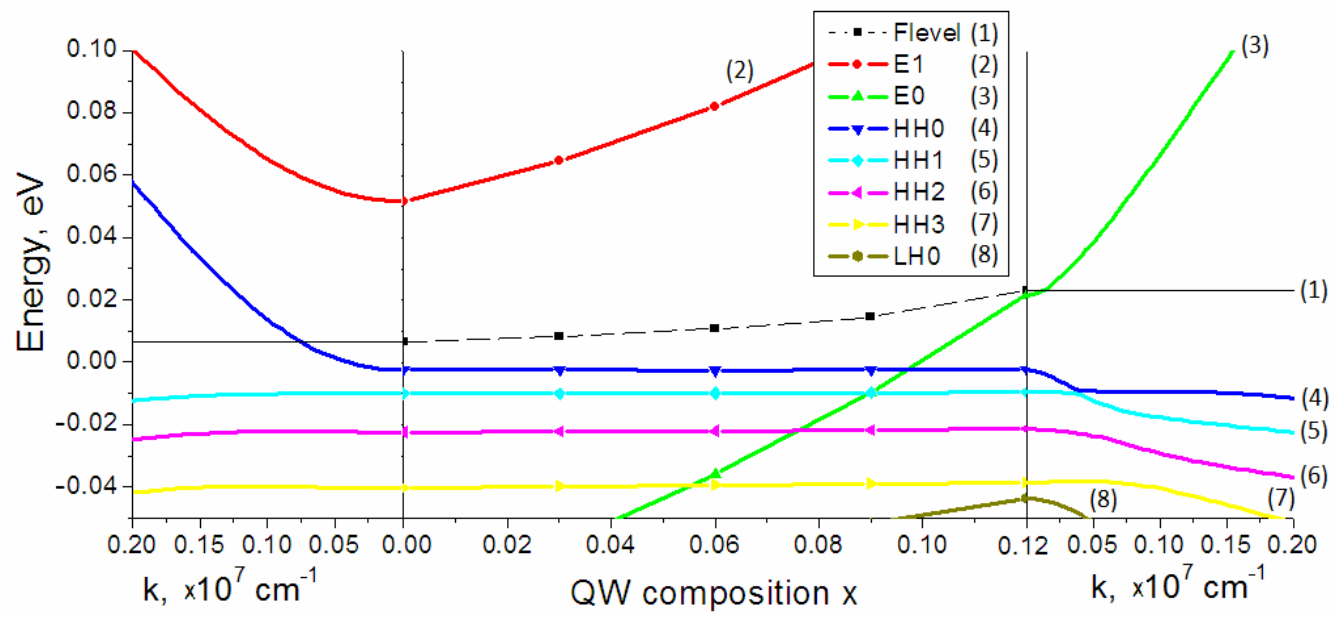

Fig. 3. Dependence of the energy spectrum and Fermi level of QW with the width $L=20 \mathrm{~nm}$ on the composition $x$. Curves on the graph are marked as follows: Fermi level - (1), E1 level - (2), E0 level - (3), HH0 level - (4), HH1 level - (5), HH2 level (6), HH3 level - (7), LH0 level - (8). 


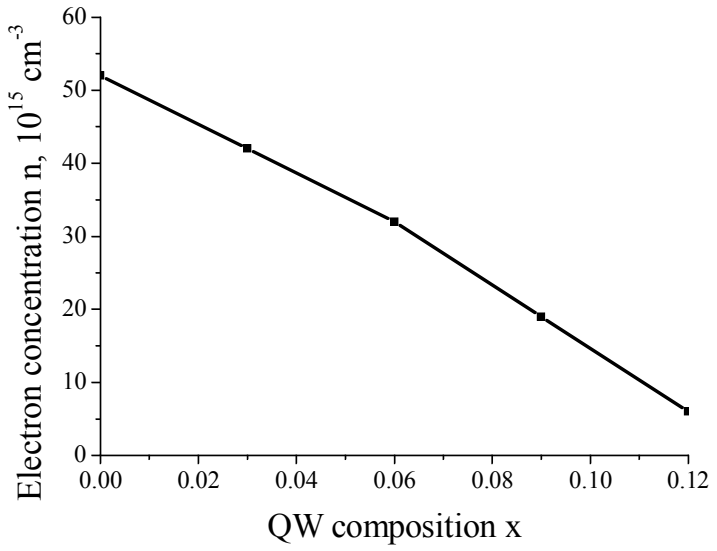

Fig. 4. Dependence of the intrinsic concentration on the QW composition $x$, well width $L=20 \mathrm{~nm}$.

The dependence of the electron concentration on the QW composition $x$ is presented in Fig. 4. The corresponding energy spectrum is presented in Fig. 3.

It should be noted that composition changes also lead to the topological phase transition. For $x=0$, the critical thickness for the $\mathrm{Hg}_{0.32} \mathrm{Cd}_{0.68} \mathrm{Te} / \mathrm{Hg}_{1-\mathrm{x}} \mathrm{Cd}_{\mathrm{x}} \mathrm{Te}$ quantum well is $L=6.7 \mathrm{~nm}$, while for higher concentrations, this thickness grows and approaches more than $L \approx 20 \mathrm{~nm}$ at $x=0.12$, which should be accounted when growing MCT QWs for $\mathrm{THz}$ bolometer detectors. This effect can be explained by stronger influence of the semiconducting barrier layers, which enhances when the well band gap decreases. The carrier concentration in the well (Fig. 4) decreases as the composition grows. This dependence is almost linear.

\section{Conclusions}

The maximal intrinsic concentration of carriers of the order of $n \sim 5 \cdot 10^{16} \mathrm{~cm}^{-3}$ can be achieved for compositions $x$ close to 0 and well widths that are greater than the critical thickness of the topological transition. For bolometer type detectors, the optimal intrinsic concentration seems to be one order lower [2].
It should be noted that the increase of the composition $x$ leads to decrease of the electron effective mass and, thus, to increase of the electron mobility, but the carrier concentration becomes smaller. Thus, for ntype samples the carrier conductivity will be influenced by two concurring trends which should be taken into account when designing QWs for THz/sub-THz detectors.

\section{References}

1. F. Sizov, V. Petriakov, V. Zabudsky, D. Krasilnikov, M. Smoliy, and S. Dvoretski, Millimeter-wave hybrid un-cooled narrow-gap hotcarrier and Schottky diodes direct detectors // Appl. Phys. Lett. 101, 082108 (2012).

2. V. Dobrovolski, F. Sizov, THz/sub-THz bolometer based on electron heating in a semiconductor waveguide // Optoelectronics Review, 18, p. 250258 (2010).

3. Jun Li and Kai Chang, Electric field driven quantum phase transition between band insulator and topological insulator // Appl. Phys. Lett. 95, 222110 (2009).

4. M. König, S. Wiedmann, C. Brüne, A. Roth, H. Buhmann, L.W. Molenkamp, Xiao-Liang Qi, ShouCheng Zhang, Quantum spin Hall insulator state in HgTe quantum wells // Science, 318 (5851), p. 766770 (2007).

5. B.A. Bernevig, T.L. Hughes, Shou-Cheng Zhang, Quantum spin Hall effect and topological phase transition in HgTe quantum wells // Science, 314 (5806), p. 1757-1761 (2006).

6. V. Latussek, Dissertation zur Erlangung des naturwissenschaftlichen Doktorgrades "Elektronische Zustande in Typ-IIIHalbleiterheterostrukturen“, Julius-MaximiliansUniversitat, Wurzburg, 2004.

7. E.G. Novik, A. Pfeuffer-Jeschke, T. Jungwirth, V. Latussek, C.R. Becker, G. Landwehr, H. Buhmann, and L.W. Molenkamp, Band structure of semimagnetic $\mathrm{Hg}_{1-\mathrm{y}} \mathrm{Mn}_{\mathrm{y}} \mathrm{Te}$ quantum wells // Phys. Rev. B, 72, 035321 (2005). 